\title{
CULTIVARES DE SOJA [Glycine max (L.) Merrill] PARA CULTIVO DE VERÃO NA REGIÃO DE LAVRAS-MG
}

\section{Soybean cultivars [Glycine max (L.) MerrilL] for summer culture in Lavras - MG region}

\author{
Fabrício de Souza Guimarães ${ }^{1}$, Pedro Milanez de Rezende ${ }^{2}$, Evaristo Mauro de Castro ${ }^{3}$, \\ Eudes de Arruda Carvalho ${ }^{4}$, Messias José Bastos de Andrade ${ }^{5}$, Everson Reis Carvalho ${ }^{6}$
}

\begin{abstract}
RESUMO
Objetivou-se avaliar cultivares de soja quanto ao rendimento de grãos e outras características agronômicas em dois anos agrícolas, em cultivo de verão (2003/2004 e 2004/2005), buscando fornecer subsídios para a escolha adequada de cultivares para a região de Lavras-MG. Utilizou-se o delineamento experimental de blocos casualizados com três repetições onde os tratamentos foram dispostos em um esquema fatorial 2 x 40, compreendendo 2 anos agrícolas (2003/04 e 2004/05) e 40 cultivares de soja. A região de Lavras/MG é propícia para o desenvolvimento da cultura de soja, visto que as cultivares apresentaram características agronômicas desejáveis durante os dois anos de experimento, com produtividade média de $2870 \mathrm{~kg}$.ha ${ }^{-1}$. As cultivares que destacaramse na média dos dois anos de cultivo foram: Vencedora (4515 kg.ha-1), Conquista (4209 kg.ha $\left.{ }^{-1}\right)$ e Monarca $\left(4121 \mathrm{~kg} \cdot \mathrm{ha}^{-1}\right)$, rendimentos esses superiores a média nacional, tendo também apresentado condições satisfatórias para a colheita mecânica.
\end{abstract}

Termos para indexação: Acamamento, produtividade.

\begin{abstract}
The purpose of this study was to evaluate soybean materials [Glycine max (L.) Merrill] for grain yield and other agronomic traits in two agricultural years during the summer in the years of (2003/04 and 2004/05) trying to provide subsidies for the right choice of the cultivars for Lavras-MG region. The experiment was evaluated in a complet block design with three replications in a factorial structure 2 x 40; 2 agricultural years (2003/04 and 2004/05) and 40 soybeans cultivars. Lavras-MG region is good for soybean culture development, because the cultivars showed desirable agronomical characteristics during the two year-experiment, the average yield was $2870 \mathrm{~kg}^{-h a^{-1}}$. The best cultivars for general standard during the two years were Vencedora (4515 kg.ha-1), Conquista (4209 kg.ha $\left.{ }^{-1}\right)$ and Monarca (4121 $\mathrm{kg} \cdot \mathrm{ha}^{-1}$ ) that also showed good conditions for the mechanical harvest.
\end{abstract}

Index terms: Cultivars, years.

(Recebido em 23 de agosto de 2006 e aprovado em 22 de fevereiro de 2007)

\section{INTRODUÇÃO}

A produtividade de uma cultura é definida pela interação entre o genótipo da planta, o ambiente de produção e o manejo. Altos rendimentos só são obtidos quando as condições ambientais são favoráveis em todos os estádios de crescimento da soja. Visando a obtenção de altos rendimentos é necessário conhecer práticas culturais compatíveis com produção econômica, aplicadas para maximizar a taxa de acúmulo de matéria seca no grão. As principais práticas de manejo que devem ser consideradas são: a semeadura na época recomendada para a região de produção; a escolha dos cultivares mais adaptados a essa região; o uso de espaçamentos e densidades adequados a esses cultivares; o monitoramento e controle das plantas daninhas, pragas e doenças e redução ao mínimo das possíveis perdas de colheita (RITCHIE et al., 1994).

Sabe-se que os fatores climáticos que condicionam o ambiente são determinantes no grau de adaptação dos indivíduos. Medeiros et al. (1991) relatam que as causas dos baixos níveis de rendimentos de grãos podem ser atribuídas ao fator de aptidão climática e edáfica da região e nível de tecnologia aplicada. De acordo com Câmara (1998a), durante o seu ciclo, a planta permanece exposta a muitos fatores externos que podem favorecer ou prejudicar a produção final.

\footnotetext{
'Engenheiro Agrônomo, Doutorando em Grandes Culturas - Departamento de Agricultura/DAG - Universidade Federal de Lavras/UFLA - Cx. P. 3037 37200-000 - Lavras, MG - fsguima@hotmail.com

²Engenheiro Agrônomo, Doutor, Professor - Departamento de Agricultura/DAG - Universidade Federal de Lavras/UFLA - Cx. P. 3037 - $37200-000$ Lavras, MG - pmrezend@ufla.br - Bolsista do CNPq

${ }^{3}$ Engenheiro Florestal, Doutor, Professor Adjunto - Departamento de Biologia/DBI- Universidade Federal de Lavras/UFLA - Cx. P. 3037 - $37200-000$ Lavras, MG - emcastro@ufla.br

${ }^{4}$ Engenheiro Agrônomo, Mestre - Departamento de Fitopatologia/DFP- Universidade Federal de Lavras/UFLA - Cx. P. 3037 - $37200-000$ - Lavras, MG eudesarruda@hotmail.com

${ }^{5}$ Engenheiro Agrônomo, Doutor - Departamento de Agricultura/DAG - Universidade Federal de Lavras/UFLA - Cx. P. 3037 - $37200-000$ - Lavras, MG mandrade@ufla.br

${ }^{6}$ Graduando em Agronomia - Departamento de Agricultura/DAG - Universidade Federal de Lavras/UFLA - Cx. P. 3037 - $37200-000$ - Lavras, MG eversonreiscarvalho@hotmail.com
} 
Existe grande variabilidade entre os cultivares com relação à sensibilidade à época de semeadura e à mudanças na região de cultivo. Por isso, são importantes os ensaios regionais de avaliação de cultivares de soja, realizados em diferentes épocas em uma mesma região (PEIXOTO et al., 2000).

A população é fator determinante para o arranjo das plantas no ambiente de produção e influencia o crescimento da soja. Dessa forma, a melhor população de plantas deve possibilitar além do alto rendimento, altura de planta e de inserção da primeira vagem adequada à colheita mecanizada e plantas que não acamem (GAUDÊNCIO et al., 1990).

O uso de populações de plantas muito acima da recomendada, além de não proporcionar acréscimos no rendimento de grãos, pode acarretar riscos de perdas por acamamento e aumento do custo de produção. Por outro lado, densidades muito baixas resultam em plantas de baixo porte, menor competição da soja com as plantas daninhas e maiores perdas na colheita (CÂMARA, 1998b).

A redução do espaçamento entre linhas, o aumento da densidade nas linhas e, principalmente, o aumento da competição intra-específica têm sido considerados responsáveis pelo aumento da altura das plantas e do índice de acamamento (MARCHIORI, 1998; MARTINS, 1999).

Em consequência da escassez de trabalhos para a região de Lavras e buscando o fornecimento de subsídios para uma escolha adequada de cultivares, objetivou-se com este trabalho avaliar cultivares de soja [Glycine max (L.) Merrill] quanto ao rendimento de grãos e outras características agronômicas, em dois anos agrícolas, em cultivo de verão (2003/2004 e 2004/2005).

\section{MATERIAL E MÉTODOS}

O experimento foi instalado em novembro de 2003 e 2004, no campo experimental da Universidade Federal de Lavras (Lavras-MG), situada à latitude de $21^{\circ} 14^{\prime}$ S, longitude $45^{\circ} 00 \mathrm{~W}$ e altitude de $918 \mathrm{~m}$, num solo Latossolo Vermelho Distroférrico típico (LVdf). O clima do município de Lavras possui duas estações definidas, seca de abril a setembro e chuvosa de outubro a março, é do tipo Cwb, conforme a classificação climática de Köeppen (ANTUNES, 1986).

A pluviosidade e temperaturas médias diárias durante os dois anos do experimento registrados em Lavras, podem ser visualizados nas Figuras 1 e 2 respectivamente.

O presente trabalho constituiu-se num plantio de cultivares de soja avaliadas em 2 anos agrícolas para cultivo de verão, utilizando um delineamento em blocos casualizados com três repetições, tem sido os tratamentos dispostos em fatorial $2 \times 40$, compreendendo; 2 anos agrícolas (2003/04 e 2004/05) e 40 cultivares de soja. As cultivares utilizadas foram: Aventis 7002, BR-9 Savana, BRS 136, BRS Carla, BRS GO (204) Goiânia, BRS GO Luziania, BRS Milena, Carrera, Confiança, Conquista, DM 339, DM Nobre, DM Vitória, DM 118, Doko, Embrapa 48, Emgopa 313, Emgopa 314, Emgopa 315, Emgopa 316, Garantia, IAC 19, IAC 21, Monarca, Monsoy 8329, Monsoy 108, Monsoy 109, Monsoy 8400, Monsoy 8411, Paiaguás, Performa, Preta, Renascença, Sambaíba, Santa Rosa, Splendor, STTE 02, Suprema, UFV 16 e Vencedora. As parcelas experimentais foram constituídas por quatro fileiras de $5 \mathrm{~m}$ de comprimento, espaçadas por $0,5 \mathrm{~m}$, utilizando-se como área útil duas fileiras centrais, com eliminação de $0,50 \mathrm{~m}$ em cada extremidade das mesmas a título de bordadura.

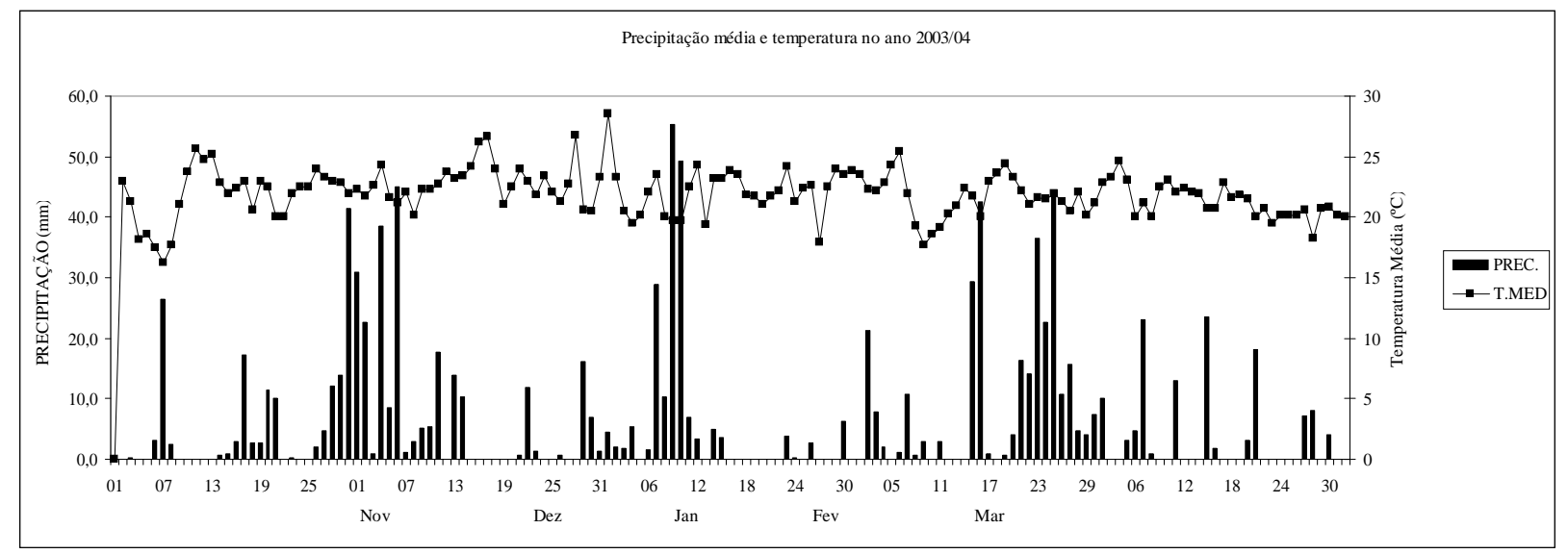

Figura 1 - Precipitação pluvial média e temperatura no ano 2003/2004, Lavras, MG.

Fonte: Estação Climatológica de Lavras, MG - UFLA. 


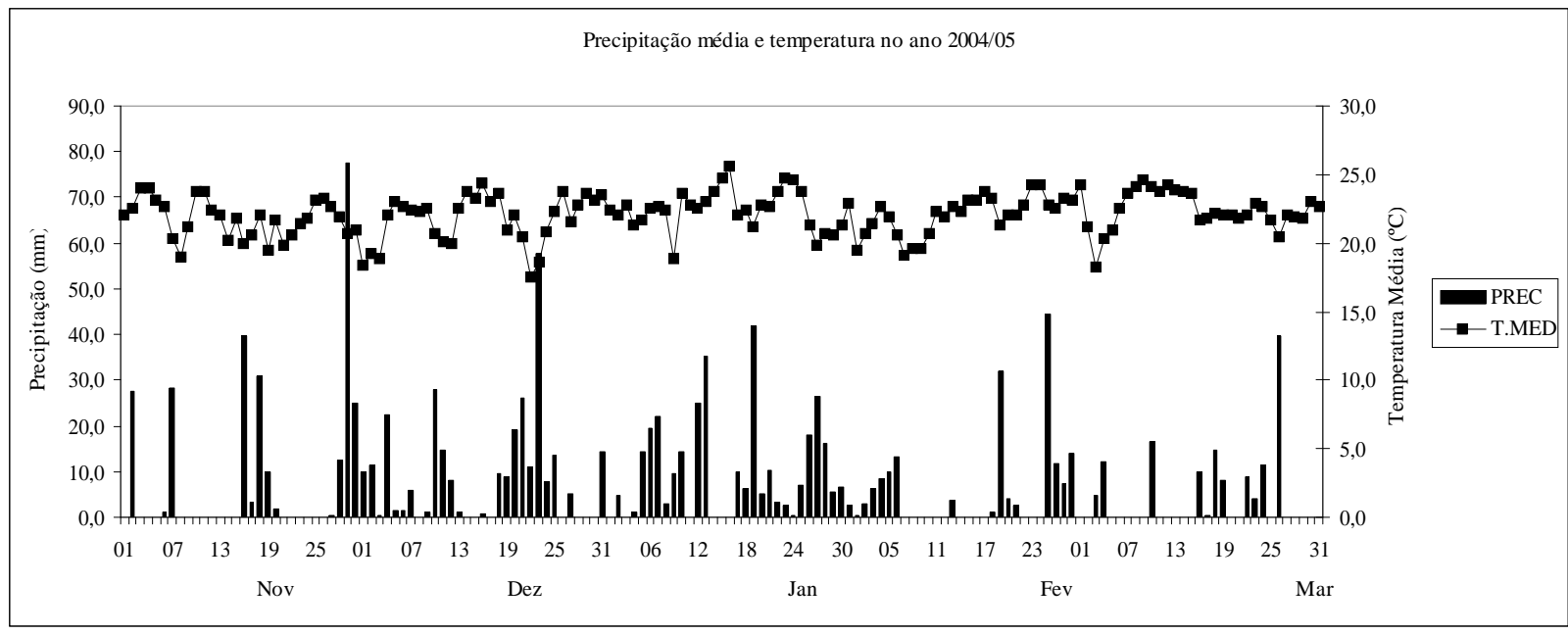

Figura 2 - Precipitação pluvial média e temperatura no ano 2004/2005, Lavras-MG. Fonte: Estação Climatológica de Lavras, MG - UFLA.

Os tratos culturais exigidos pela cultura foram realizados uniformemente em todas as parcelas experimentais, sendo utilizado uma adubação de $120 \mathrm{~kg} \cdot \mathrm{ha}^{-1}$ de $\mathrm{P}_{2} \mathrm{O}_{5}$ e 60 kg.ha ${ }^{-1}$ de $\mathrm{K}_{2} \mathrm{O}$, distribuídos e incorporados manualmente aos sulcos de semeadura e inoculação das sementes na proporção de $250 \mathrm{~g}$ de inoculante para $50 \mathrm{~kg}$ de sementes.

O comportamento da cultura foi avaliado no estádio $\mathrm{R} 8$ por meio de amostragens para determinação de rendimento de grãos ( $\left.\mathrm{kg} \cdot \mathrm{ha}^{-1}\right)$, altura da planta, ao qual utilizou-se a análise de variância, com o auxílio do programa computacional SISVAR, desenvolvido por Ferreira (2006), empregando-se o teste de Scott-Knott ao nível de 5\% de probabilidade para agrupamento de médias. Para a característica acamamento, utilizou-se um teste não paramétrico (Teste de Friedman), por causa da sua forma de avaliação.

\section{RESULTADOS E DISCUSSÃO}

\section{Rendimentos de grãos}

Observa-se para o ano de 2003/04 uma variação de 1105 a $4270 \mathrm{~kg}$.ha, obtida com as cultivares BRS Carla e Vencedora, respectivamente. Na média geral anual esses valores foram de $2121 \mathrm{~kg}$.ha para 2003/04 e $3619 \mathrm{~kg}$.ha para o ano de 2004/05 conforme indica a Tabela 1.

Analisando separadamente as cultivares dentro de cada ano, podemos verificar que no ano de 2003/04 as cultivares que mais se destacaram foram Vencedora (4270 kg.ha) e Conquista (3725 kg.ha), que proporcionaram um aumento significativo, de 286 e $237 \%$, em relação ao de menor rendimento, BRS Carla, já relatado anteriormente. No caso do ano de 2004/05, as cultivares que mais se destacaram foram Monarca (5459 kg.ha), Sambaíba (4785 kg.ha), Vencedora (4759 kg.ha), Conquista (4693 kg.ha), Emgopa 314 (4445 kg.ha) e BR-9 Savana (4405 kg.ha). Comparando, desta vez, as cultivares em relação aos anos, verificou-se que a maioria delas apresentou maiores rendimentos de grãos em 2004/05, exceto BRS 136, BRS GO Goiânia, Confiança, Doko, Embrapa 48, Emgopa 315, Emgopa 316 e Vencedora, que não apresentaram comportamento diferenciado nos dois anos de cultivo.

Na média dos dois anos, o destaque é observado para as cultivares Vencedora (4515 kg.ha), Conquista (4209 kg.ha) e Monarca (4121 kg.ha) que apresentaram rendimentos superiores à média nacional de $2.820 \mathrm{~kg}$.ha para o ano de 2004/05. É importante ressaltar que entre as cultivares citadas acima, apenas a Vencedora apresentou mesmo comportamento independentemente do ano, mantendo, assim, maior estabilidade de produção característica esta procurada nos cultivares de soja.

O rendimento de grãos é muito influenciado por vários fatores ambientais, como: umidade, temperatura e fotoperíodo que variam com as diferentes épocas do ano. Altos rendimentos podem ser obtidos quando as condições relatadas anteriormente estão em todos os estádios de desenvolvimento.

De acordo com Evans (1993), o potencial de rendimento de grãos pode ser definido como a produção de uma cultivar no ambiente ao qual está adaptada, sem limitações edafoclimáticas e nutricionais, livre da ação de pragas e doenças e com os demais estresses efetivamente controlados. Dentro desse contexto, a ação de algumas doenças como a ferrugem, mesmo com o controle sendo realizado, pode levar a uma diminuição nas produtividades 
observadas em caso de uma maior severidade da doença, fato que ocorreu no ano de 2003/04. No caso de 2004/05 esse ataque foi menos intenso, dando mais chance para um maior desenvolvimento das plantas.

Tabela 1 - Valores médios de produtividade (kg/ha) das diferentes cultivares de soja nos anos agrícolas 2003/04 e 2004/05, UFLA, Lavras -MG

\begin{tabular}{|c|c|c|c|}
\hline \multirow{2}{*}{ Cultivares } & \multicolumn{2}{|c|}{ Ano Agrícola } & \multirow{2}{*}{${ }^{1}$ Médias } \\
\hline & $2003 / 04$ & $2004 / 05$ & \\
\hline Aventis 7002 & $1880 \mathrm{~B} \mathrm{c}$ & $2833 \mathrm{~A} \mathrm{c}$ & $2357 \mathrm{~d}$ \\
\hline BR-9 Savana & $2476 \mathrm{~B} \mathrm{~b}$ & $4405 \mathrm{~A} \mathrm{a}$ & $3441 \mathrm{~b}$ \\
\hline BRS 136 & $2494 \mathrm{~A} \mathrm{~b}$ & $3153 \mathrm{~A} \mathrm{c}$ & $2824 \mathrm{c}$ \\
\hline BRS Carla & $1105 \mathrm{~B} \mathrm{c}$ & $4057 \mathrm{~A} \mathrm{~b}$ & $2581 \mathrm{~d}$ \\
\hline BRS GO (204) Goiania & $2045 \mathrm{~A} \mathrm{c}$ & $1781 \mathrm{~A} \mathrm{~d}$ & $1913 d$ \\
\hline BRS GO Luziania & $1770 \mathrm{~B} \mathrm{c}$ & $3811 \mathrm{~A} \mathrm{~b}$ & $2791 \mathrm{c}$ \\
\hline BRS Milena & $1796 \mathrm{~B} \mathrm{c}$ & $3821 \mathrm{~A} \mathrm{~b}$ & $2809 \mathrm{c}$ \\
\hline Carrera & $1809 \mathrm{~B} \mathrm{c}$ & 2969 A c & $2389 \mathrm{~d}$ \\
\hline Confiança & $1733 \mathrm{~A} \mathrm{c}$ & $2650 \mathrm{~A} \mathrm{c}$ & $2192 \mathrm{~d}$ \\
\hline Conquista & $3725 \mathrm{~B}$ a & $4693 \mathrm{~A} \mathrm{a}$ & $4209 \mathrm{a}$ \\
\hline DM 339 & $2601 \mathrm{~A} \mathrm{~b}$ & $3434 \mathrm{~A} \mathrm{c}$ & $3018 \mathrm{c}$ \\
\hline DM Nobre & $1335 \mathrm{~B} \mathrm{c}$ & $3848 \mathrm{~A} \mathrm{~b}$ & $2591 \mathrm{~d}$ \\
\hline DM Vitória & $2326 \mathrm{~B} \mathrm{~b}$ & $4272 \mathrm{~A} \mathrm{~b}$ & $3299 \mathrm{~b}$ \\
\hline DM 118 & $2461 \mathrm{~B} \mathrm{~b}$ & 3449 A c & $2955 \mathrm{c}$ \\
\hline DOKO & $2054 \mathrm{~A} \mathrm{c}$ & $1938 \mathrm{Ad}$ & $1996 \mathrm{~d}$ \\
\hline Embrapa 48 & $2133 \mathrm{~A} \mathrm{c}$ & $2730 \mathrm{~A} \mathrm{c}$ & $2431 \mathrm{~d}$ \\
\hline Emgopa 313 & $2351 \mathrm{~B} \mathrm{~b}$ & $3702 \mathrm{~A} \mathrm{~b}$ & $3026 \mathrm{c}$ \\
\hline Emgopa 314 & $2769 \mathrm{~B} \mathrm{~b}$ & $4445 \mathrm{~A} \mathrm{a}$ & $3607 \mathrm{~b}$ \\
\hline Emgopa 315 & $2419 \mathrm{~A} \mathrm{~b}$ & $3285 \mathrm{~A} \mathrm{c}$ & $2852 \mathrm{c}$ \\
\hline Emgopa 316 & $2167 \mathrm{~A} \mathrm{c}$ & $2779 \mathrm{~A} \mathrm{c}$ & $2473 \mathrm{~d}$ \\
\hline Garantia & $2200 \mathrm{~B} \mathrm{c}$ & $4142 \mathrm{~A} \mathrm{~b}$ & $3171 \mathrm{~b}$ \\
\hline IAC 19 & 2014 B c & $4316 \mathrm{~A} \mathrm{~b}$ & $3165 \mathrm{~b}$ \\
\hline IAC 21 & $2286 \mathrm{~B} \mathrm{~b}$ & $3856 \mathrm{~A} \mathrm{~b}$ & $3071 \mathrm{c}$ \\
\hline Monarca & $2782 \mathrm{~B} \mathrm{~b}$ & $5459 \mathrm{~A} \mathrm{a}$ & $4121 \mathrm{a}$ \\
\hline Monsoy 8329 & 1925 B c & $3476 \mathrm{~A} \mathrm{c}$ & $2701 \mathrm{c}$ \\
\hline Monsoy 108 & $1269 \mathrm{~B} \mathrm{c}$ & $3819 \mathrm{~A} \mathrm{~b}$ & $2544 \mathrm{~d}$ \\
\hline Monsoy 109 & $2497 \mathrm{~B} \mathrm{~b}$ & $4076 \mathrm{~A} \mathrm{~b}$ & $3286 \mathrm{~b}$ \\
\hline Monsoy 8400 & $1715 \mathrm{~B} \mathrm{c}$ & $3555 \mathrm{~A} \mathrm{c}$ & $2635 \mathrm{c}$ \\
\hline Monsoy 8411 & $1691 \mathrm{~B} \mathrm{c}$ & $3181 \mathrm{~A} \mathrm{c}$ & $2436 \mathrm{~d}$ \\
\hline Paiaguás & $2356 \mathrm{~B} \mathrm{~b}$ & $3891 \mathrm{~A} \mathrm{~b}$ & $3124 \mathrm{~b}$ \\
\hline Performa & $2041 \mathrm{~B} \mathrm{c}$ & $3865 \mathrm{~A} \mathrm{~b}$ & $2953 c$ \\
\hline Preta & 2064 B c & 3997 A b & $3031 \mathrm{c}$ \\
\hline Renascença & $1510 \mathrm{~B} \mathrm{c}$ & 2923 A c & $2217 \mathrm{~d}$ \\
\hline Sambaíba & $2498 \mathrm{~B} \mathrm{~b}$ & $4785 \mathrm{~A} \mathrm{a}$ & $3642 \mathrm{~b}$ \\
\hline Santa Rosa & $1531 \mathrm{~B} \mathrm{c}$ & 3549 A c & $2540 \mathrm{~d}$ \\
\hline Splendor & 1348 B c & $3290 \mathrm{~A} \mathrm{c}$ & $2319 d$ \\
\hline STTE 02 & $1703 \mathrm{~B} \mathrm{c}$ & $3847 \mathrm{~A} \mathrm{~b}$ & $2775 \mathrm{c}$ \\
\hline Suprema & 1902 B c & $2857 \mathrm{~A} \mathrm{c}$ & $2379 d$ \\
\hline UFV 16 & $1780 \mathrm{~B} \mathrm{c}$ & $3061 \mathrm{~A} \mathrm{c}$ & $2420 \mathrm{~d}$ \\
\hline Vencedora & $4270 \mathrm{~A} \mathrm{a}$ & $4759 \mathrm{~A} \mathrm{a}$ & $4515 \mathrm{a}$ \\
\hline${ }^{2}$ Médias & $2121 \mathrm{~B}$ & $3619 \mathrm{~A}$ & \\
\hline
\end{tabular}

Ciênc. agrotec., Lavras, v. 32, n. 4, p. 1099-1106, jul./ago., 2008 
As cultivares que apresentaram melhores rendimentos no presente estudo coincidem com os mais utilizados pelos agricultores no estado de Minas Gerais, o que demonstra a capacidade de adaptação desses genótipos às mais variadas condições ambientais.

\section{Altura de planta}

A altura de planta, apresentou uma variação de 64 a $140 \mathrm{~cm}$ para o ano agrícola 2003/04 e de 64 a $112 \mathrm{~cm}$ no agrícola 2004/05, sendo consideradas satisfatórias para a colheita mecânica.

Pode ser observado, para o ano de 2003/04, que a cultivar DM Vitória foi a que apresentou maior altura de plantas pelo teste de Scott-Konott com um nível de significância de 5\%. Esta cultivar foi seguida de um segundo grupo formado pelas cultivares BRS Milena, Emgopa 314, Emgopa 313, Monsoy 8329, DM Nobre, Paiaguás, Garantia, Monsoy 109, DM 339, Sambaíba, Monsoy 108, Monarca e Monsoy 8411. A cultivar que apresentou a menor altura de plantas foi a Embrapa 48. $\mathrm{Na}$ Tabela 2, podemos observar também as alturas médias de plantas das diferentes cultivares para o ano de 2004/05. Para este ano, as maiores alturas médias de plantas foram das cultivares DM Vitória, Emgopa 314, DM Nobre, Monsoy 108, Garantia e Suprema. As menores alturas médias de plantas foram das cultivares BRS 136, BRS GO (204) Goiânia, BRS GO Luziania, Embrapa 48 e SITTE 02.

Em média houve um aumento significativo de $16,48 \%(14,43 \mathrm{~cm})$ na altura da planta do ano de 2003/ 04 para o ano de 2004/05. Em relação aos dados referentes à média dos dois anos agrícolas, a cultivar DM Vitória foi também a responsável pela maior altura observada.
A altura de planta é característica fundamental na determinação da cultivar a ser introduzido em uma região, uma vez que se relaciona com o rendimento de grãos, o controle de plantas daninhas e as perdas durante a colheita mecanizada. As variações na altura de plantas podem ser influenciadas por época de semeadura, espaçamento de plantas entre e dentro das fileiras, suprimento de umidade, temperatura, fertilidade do solo e outras condições gerais do meio ambiente. Dependendo da resposta fotoperiódica da cultivar, a planta pode ter altura reduzida, sendo consideradas adequadas à mecanização da colheita plantas com altura entre 60 e $120 \mathrm{~cm}$, conforme relatam Cartter \& Hartwig (1967).

\section{Acamamento}

Essa característica assume importante papel na seleção de cultivares, visto que poderá provocar perdas no processo de colheita mecanizada, juntamente com altura de planta e inserção do primeiro legume. Verificou-se notas compreendidas entre 1 e 4 para o ano de 2003/04, e as cultivares que obtiveram o maior índice de acamamento foram a Splendor e DM Nobre. No ano de 2004/05, as notas variaram de 1 a 2 , não havendo diferença significativa entre as cultivares de acordo com a Tabela 3 .

O acamamento é uma característica muito influenciada pelo tipo de solo e pelas condições de desenvolvimento da planta. Em geral, as plantas de soja sofrem maior acamamento em solos férteis e pesados, com umidade abundante, do que em solos leves e arenosos. Outro ponto a considerar refere-se à altura de planta, normalmente plantas mais altas poderão proporcionar um maior índice de acamamento dado ao fato de apresentarem caule mais finos, ficando mais sujeitas a ação dos ventos. 
Tabela 2 - Valores médios da altura da planta das diferentes cultivares de soja nos anos agrícolas 2003/04 e 2004/05, UFLA. Lavras-MG.

\begin{tabular}{|c|c|c|c|}
\hline \multirow{2}{*}{ Cultivares } & \multicolumn{2}{|c|}{ Ano Agrícola } & \multirow{2}{*}{${ }^{1}$ Médias } \\
\hline & $2003 / 04$ & $2004 / 05$ & \\
\hline Aventis 7002 & $96,00 \mathrm{~A} \mathrm{c}$ & $93,00 \mathrm{Ab}$ & $94,50 \mathrm{~d}$ \\
\hline BR-9 Savana & $103,33 \mathrm{Ac}$ & $90,26 \mathrm{~A} \mathrm{~b}$ & $96,80 \mathrm{~d}$ \\
\hline BRS 136 & $94,00 \mathrm{~A} \mathrm{c}$ & $71,46 \mathrm{~B} \mathrm{~d}$ & $82,73 \mathrm{e}$ \\
\hline BRS Carla & $91,66 \mathrm{~A} \mathrm{c}$ & $90,13 \mathrm{~A} \mathrm{~b}$ & $90,90 \mathrm{~d}$ \\
\hline BRS GO (204) Goiania & 99,66 A c & $65,46 \mathrm{~B} \mathrm{~d}$ & $82,57 \mathrm{e}$ \\
\hline BRS GO Luziania & $97,00 \mathrm{~A} \mathrm{c}$ & $74,53 \mathrm{~B} \mathrm{~d}$ & $85,77 \mathrm{e}$ \\
\hline BRS Milena & 124,66 A b & 94,06 B b & $109,36 \mathrm{c}$ \\
\hline Carrera & $94,33 \mathrm{~A} \mathrm{c}$ & $82,66 \mathrm{Ac}$ & $88,50 \mathrm{~d}$ \\
\hline Confiança & $93,66 \mathrm{~A} \mathrm{c}$ & $81,53 \mathrm{~A} \mathrm{c}$ & $87,60 \mathrm{~d}$ \\
\hline Conquista & $79,10 \mathrm{~A} \mathrm{~d}$ & $79,13 \mathrm{~A} \mathrm{c}$ & $79,12 \mathrm{e}$ \\
\hline DM 339 & $114,33 \mathrm{~A} \mathrm{~b}$ & $95,80 \mathrm{~B} \mathrm{~b}$ & $105,07 \mathrm{c}$ \\
\hline DM Nobre & $119,00 \mathrm{~A} \mathrm{~b}$ & $108,20 \mathrm{~A} \mathrm{a}$ & $113,60 \mathrm{~b}$ \\
\hline DM Vitória & $140,33 \mathrm{~A} \mathrm{a}$ & $112,93 \mathrm{~B} \mathrm{a}$ & $126,63 \mathrm{a}$ \\
\hline DM 118 & $101,00 \mathrm{~A} \mathrm{c}$ & $78,33 \mathrm{~B} \mathrm{c}$ & $89,66 \mathrm{~d}$ \\
\hline DOKO & $96,66 \mathrm{~A} \mathrm{c}$ & $88,60 \mathrm{Ab}$ & $92,63 \mathrm{~d}$ \\
\hline Embrapa 48 & $64,66 \mathrm{~A} \mathrm{e}$ & $64,53 \mathrm{~A} \mathrm{~d}$ & $64,60 \mathrm{f}$ \\
\hline Emgopa 313 & $122,00 \mathrm{~A} \mathrm{~b}$ & $96,93 \mathrm{~B} \mathrm{~b}$ & $109,46 \mathrm{c}$ \\
\hline Emgopa 314 & $122,66 \mathrm{~A} \mathrm{~b}$ & $110,46 \mathrm{~A} \mathrm{a}$ & $116,56 \mathrm{~b}$ \\
\hline Emgopa 315 & $97,00 \mathrm{~A} \mathrm{c}$ & $83,13 \mathrm{~A} \mathrm{c}$ & $90,07 \mathrm{~d}$ \\
\hline Emgopa 316 & $96,00 \mathrm{~A} \mathrm{c}$ & $91,33 \mathrm{~A} \mathrm{~b}$ & $93,67 \mathrm{~d}$ \\
\hline Garantia & $115,33 \mathrm{~A} \mathrm{~b}$ & $101,93 \mathrm{~A} \mathrm{a}$ & $108,63 \mathrm{c}$ \\
\hline IAC 19 & $102,00 \mathrm{~A} \mathrm{c}$ & $82,73 \mathrm{~B} \mathrm{c}$ & $92,37 \mathrm{~d}$ \\
\hline IAC 21 & $100,66 \mathrm{Ac}$ & $82,26 \mathrm{~B} \mathrm{c}$ & $91,47 \mathrm{~d}$ \\
\hline Monarca & $107,33 \mathrm{~A} \mathrm{~b}$ & $93,73 \mathrm{~A} \mathrm{~b}$ & $100,53 \mathrm{c}$ \\
\hline Monsoy 8329 & $119,00 \mathrm{~A} \mathrm{~b}$ & 84,73 B c & $101,86 \mathrm{c}$ \\
\hline Monsoy 108 & $110,00 \mathrm{~A} \mathrm{~b}$ & $102,53 \mathrm{~A} \mathrm{a}$ & $106,26 \mathrm{c}$ \\
\hline Monsoy 109 & $114,66 \mathrm{~A} \mathrm{~b}$ & $93,00 \mathrm{~B} \mathrm{~b}$ & $103,83 \mathrm{c}$ \\
\hline Monsoy 8400 & $101,33 \mathrm{~A} \mathrm{c}$ & $86,33 \mathrm{~B} \mathrm{c}$ & $93,83 \mathrm{~d}$ \\
\hline Monsoy 8411 & $107,00 \mathrm{~A} \mathrm{~b}$ & $87,06 \mathrm{~B} \mathrm{c}$ & $97,03 \mathrm{~d}$ \\
\hline Paiaguás & $117,33 \mathrm{~A} \mathrm{~b}$ & $92,46 \mathrm{~B} \mathrm{~b}$ & $104,90 \mathrm{c}$ \\
\hline Performa & $86,00 \mathrm{Ad}$ & $84,53 \mathrm{~A} \mathrm{c}$ & $85,27 \mathrm{e}$ \\
\hline Preta & $83,33 \mathrm{Ad}$ & $77,06 \mathrm{~A} \mathrm{c}$ & $80,20 \mathrm{e}$ \\
\hline Renascença & $86,00 \mathrm{Ad}$ & $77,00 \mathrm{~A} \mathrm{c}$ & $81,50 \mathrm{e}$ \\
\hline Sambaíba & $112,00 \mathrm{~A} \mathrm{~b}$ & $95,26 \mathrm{~B} \mathrm{~b}$ & $103,63 \mathrm{c}$ \\
\hline Santa Rosa & $102,00 \mathrm{~A} \mathrm{c}$ & $79,66 \mathrm{~B} \mathrm{c}$ & $90,83 \mathrm{~d}$ \\
\hline Splendor & $99,66 \mathrm{Ac}$ & $92,00 \mathrm{~A} \mathrm{~b}$ & $95,83 \mathrm{~d}$ \\
\hline SITTE 02 & $83,66 \mathrm{~A} \mathrm{~d}$ & $68,53 \mathrm{~B} \mathrm{~d}$ & $76,10 \mathrm{e}$ \\
\hline Suprema & $102,66 \mathrm{~A} \mathrm{c}$ & $101,16 \mathrm{~A} \mathrm{a}$ & $101,91 \mathrm{c}$ \\
\hline UFV 16 & $102,66 \mathrm{~A} \mathrm{c}$ & $82,60 \mathrm{~B} \mathrm{c}$ & $92,63 \mathrm{~d}$ \\
\hline Vencedora & $79,50 \mathrm{~A} \mathrm{~d}$ & $84,93 \mathrm{~A} \mathrm{c}$ & $82,22 \mathrm{e}$ \\
\hline${ }^{2}$ Médias & $101,98 \mathrm{~A}$ & $87,55 \mathrm{~B}$ & \\
\hline
\end{tabular}

1- Médias seguidas de mesma letra minúscula, na coluna, não diferem entre si pelo teste de Scott-Knott com um nível nominal de significância de $5 \%$.

2 - Médias seguidas de mesma letra maiúscula, na linha, não diferem entre si pelo teste F com um nível nominal de significância de 5\%. 
Tabela 3 - Valores médios de nota de acamamento das plantas e totais de ordenação (Postos) das diferentes cultivares de soja em função do ano estudado, de acordo com o Teste de Friedman, obtidos no ensaio de cultivares, ano agrícola 2003/04 e 2004/05, UFLA, Lavras-MG.

\begin{tabular}{|c|c|c|c|c|}
\hline \multirow{3}{*}{ Cultivares } & \multicolumn{4}{|c|}{ Ano Agrícola } \\
\hline & \multicolumn{2}{|c|}{$2003 / 04$} & \multicolumn{2}{|c|}{$2004 / 05$} \\
\hline & Nota média & ${ }^{1}$ Postos & Nota média & ${ }^{1}$ Postos \\
\hline Aventis 7002 & 1 & $32,0 \mathrm{fg}$ & 1 & $48,0 \mathrm{a}$ \\
\hline BR-9 Savana & 2 & 43,0 efg & 1 & $67,5 \mathrm{a}$ \\
\hline BRS 136 & 1 & $31,5 \mathrm{fg}$ & 1 & $48,0 \mathrm{a}$ \\
\hline BRS Carla & 2 & 44,5 efg & 1 & $67,5 \mathrm{a}$ \\
\hline BRS GO (204) Goiania & 3 & 81,5 abcd & 1 & $67,5 \mathrm{a}$ \\
\hline BRS GO Luziania & 1 & $31,5 \mathrm{fg}$ & 1 & $67,5 \mathrm{a}$ \\
\hline BRS Milena & 2 & 67,5 cde & 1 & $48,0 \mathrm{a}$ \\
\hline Carrera & 1 & $19,0 \mathrm{~g}$ & 1 & $48,0 \mathrm{a}$ \\
\hline Confiança & 2 & 44,5 efg & 1 & $48,0 \mathrm{a}$ \\
\hline Conquista & 1 & $19,0 \mathrm{~g}$ & 1 & $48,0 \mathrm{a}$ \\
\hline DM 339 & 3 & 79,0 abcd & 2 & $107,0 \mathrm{a}$ \\
\hline DM Nobre & 4 & $107,5 \mathrm{a}$ & 2 & $107,0 \mathrm{a}$ \\
\hline DM Vitória & 3 & 81,5 abcd & 2 & $90,5 \mathrm{a}$ \\
\hline DM 118 & 1 & $32,0 \mathrm{fg}$ & 1 & $48,0 \mathrm{a}$ \\
\hline DOKO & 3 & $93,0 \mathrm{abc}$ & 1 & $48,0 \mathrm{a}$ \\
\hline Embrapa 48 & 1 & $19,0 \mathrm{~g}$ & 1 & $48,0 \mathrm{a}$ \\
\hline Emgopa 313 & 3 & $81,5 \mathrm{abcd}$ & 1 & $67,5 \mathrm{a}$ \\
\hline Emgopa 314 & 3 & $88,5 \mathrm{abc}$ & 2 & $113,5 \mathrm{a}$ \\
\hline Emgopa 315 & 2 & 67,0 cde & 1 & $48,0 \mathrm{a}$ \\
\hline Emgopa 316 & 2 & $55,5 \mathrm{def}$ & 1 & $48,0 \mathrm{a}$ \\
\hline Garantia & 2 & 69,0 bcde & 1 & $48,0 \mathrm{a}$ \\
\hline IAC 19 & 2 & 67,0 cde & 1 & $48,0 \mathrm{a}$ \\
\hline IAC 21 & 3 & 81,5 abcd & 1 & $48,0 \mathrm{a}$ \\
\hline Monarca & 2 & 67,0 cde & 1 & $48,0 \mathrm{a}$ \\
\hline Monsoy 8329 & 3 & $100,5 \mathrm{ab}$ & 1 & $48,0 \mathrm{a}$ \\
\hline Monsoy 108 & 2 & $44,5 \mathrm{efg}$ & 2 & $87,5 \mathrm{a}$ \\
\hline Monsoy 109 & 1 & $32,0 \mathrm{fg}$ & 1 & $48,0 \mathrm{a}$ \\
\hline Monsoy 8400 & 3 & $100,5 \mathrm{ab}$ & 2 & $87,5 \mathrm{a}$ \\
\hline Monsoy 8411 & 3 & $100,5 \mathrm{ab}$ & 1 & $68,0 \mathrm{a}$ \\
\hline Paiaguás & 3 & $93,0 \mathrm{abc}$ & 2 & $107,0 \mathrm{a}$ \\
\hline Performa & 3 & $88,0 \mathrm{abc}$ & 1 & $48,0 \mathrm{a}$ \\
\hline Preta & 2 & 43,0 efg & 1 & $48,0 \mathrm{a}$ \\
\hline Renascença & 1 & $19,0 \mathrm{~g}$ & 1 & $67,5 \mathrm{a}$ \\
\hline Sambaíba & 3 & $100,5 \mathrm{ab}$ & 1 & $48,0 \mathrm{a}$ \\
\hline Santa Rosa & 2 & 42,5 efg & 1 & $67,5 \mathrm{a}$ \\
\hline Splendor & 4 & $109,0 \mathrm{a}$ & 1 & $48,0 \mathrm{a}$ \\
\hline STTE 02 & 2 & 67,0 cde & 1 & $48,0 \mathrm{a}$ \\
\hline Suprema & 1 & $19,0 \mathrm{~g}$ & 1 & $48,0 \mathrm{a}$ \\
\hline UFV 16 & 3 & 79,0 abcd & 1 & $67,5 \mathrm{a}$ \\
\hline Vencedora & 1 & $19,0 \mathrm{~g}$ & 1 & $48,0 \mathrm{a}$ \\
\hline \multirow{2}{*}{ Chi-quadrado } & Calculado & $81,694 *$ & & $38,566 \mathrm{~ns}$ \\
\hline & Tabelado (78 gl) & 54,570 & & 54,570 \\
\hline
\end{tabular}

1 - Médias seguidas de mesma letra minúscula, na coluna, não diferem entre si pelo teste de Friedman com um nível nominal de significância de 5\%. 


\section{CONCLUSÕES}

A região de Lavras/MG é propícia para o desenvolvimento da cultura de soja, visto que as cultivares apresentaram características agronômicas desejáveis durante os dois anos de experimento, com produtividade média de $2870 \mathrm{~kg}$.ha.

As cultivares que mais se destacaram em média geral dos anos utilizados foram Vencedora (4515 kg.ha), Conquista (4209 kg.ha) e Monarca (4121 kg.ha) que também apresentaram condições satisfatórias para a colheita mecânica.

\section{REFERÊNCIAS BIBLIOGRÁFICAS}

ANTUNES, F. Z. Caracterização climática do estado de Minas Gerais. Informe Agropecuário, Belo Horizonte, v. 12, n. 138, p. 9-13, jun. 1986 .

CÂMARA, G. M. de S. Desempenho produtivo dos cultivares de soja IAC-12, IAC-17 e IAC-19 em três épocas de semeadura e cinco densidades de plantas. 1998. $165 \mathrm{f}$. Tese (Livre Docência) - Escola Superior de Agricultura "Luiz de Queiroz”, Universidade de São Paulo, Piracicaba, 1998a.

CÂMARA, G. M. de S. Ecofisiologia da soja e rendimento. In: _. Soja: tecnologia da produção. Piracicaba: ESALQ-USP, 1998b. p. 256-577, 293 p.

CARTTER, J. L.; HARTWIG, E. E. The management of soybean. In: NORMAN, A. G. (Ed.). The soybean. New York: [s.n.], 1967. p. 162-221.

EVANS, L. T. Crop evolution, adaptation and yield. Cambridge: Cambridge University, 1993. 500 p.

FERREIRA, D. F. Sisvar - Sistema de Análise de Variância Viçosa: UFV, 2006.
GAUDÊNCIO, C.; GAZZIERO, D. L. P.; JASTER, F.; GARCIA, A.; WOBETO, C. População de plantas de soja no sistema de semeadura direta para o centro-sul do Estado do Paraná. Londrina: Centro Nacional de Pesquisa de Soja, 1990. (Comunicado técnico, 47).

MARCHIORI, L. F. S. Desempenho vegetativo e produtivo de três cultivares de soja em cinco densidades populacionais nas épocas normal e safrinha. 1999. 55 f. Dissertação (Mestrado em Fitotecnia) - Escola Superior de Agricultura 'Luís de Queiroz', Universidade de São Paulo, Piracicaba, 1998.

MARTINS, M. C. Desempenho produtivo de três cultivares de soja em duas épocas de semeadura e em cinco densidades de plantas. 1999. 84 f. Dissertação (Mestrado em Fitotecnia) - Escola Superior de Agricultura 'Luís de Queiroz', Universidade de São Paulo, Piracicaba, 1999.

MEDEIROS, S. L. P.; WESTHPHALEN, S. L.; MATZENAUER, R.; BERGAMASCHI, H. Relações entre evapotranspiração e rendimento de grãos de milho. Pesquisa Agropecuária Brasileira, Brasília, v. 26, n. 1, p. 1-10, jan. 1991.

PEIXOTO, C. P.; CÂMARA, G. M. S.; MARTINS, M. C.; MARCHIORI, L. F. S.; GUERZONI, R. A.; MATTIAZZI, P. Época de semeadura e densidade de plantas de soja: I. componentes da produção e rendimento de grãos. Scientia Agrícola, Piracicaba, v. 57, n. 1, p. 89-96, 2000.

RITCHIE, S. W.; HANWAY, J. J.; THOMPSON, H. E.; BENSON, G. O. How a soybean plant develops. Ames: Iowa State University of Science and ThechnologyCooperative Extension Service, 1994. 20 p. (Special report, 53) 